\title{
Effect of Anodic Alumina Oxide Pore Diameter on the Crystallization of Poly(butylene adipate)
}

Xiaoli Sun, Qunqun Fang, Huihui Li, Zhongjie Ren, Shouke Yan*

State Key Laboratory of Chemical Resource Engineering, Beijing University of Chemical

Technology, Beijing 100029, China (skyan@mail.buct.edu.cn) 

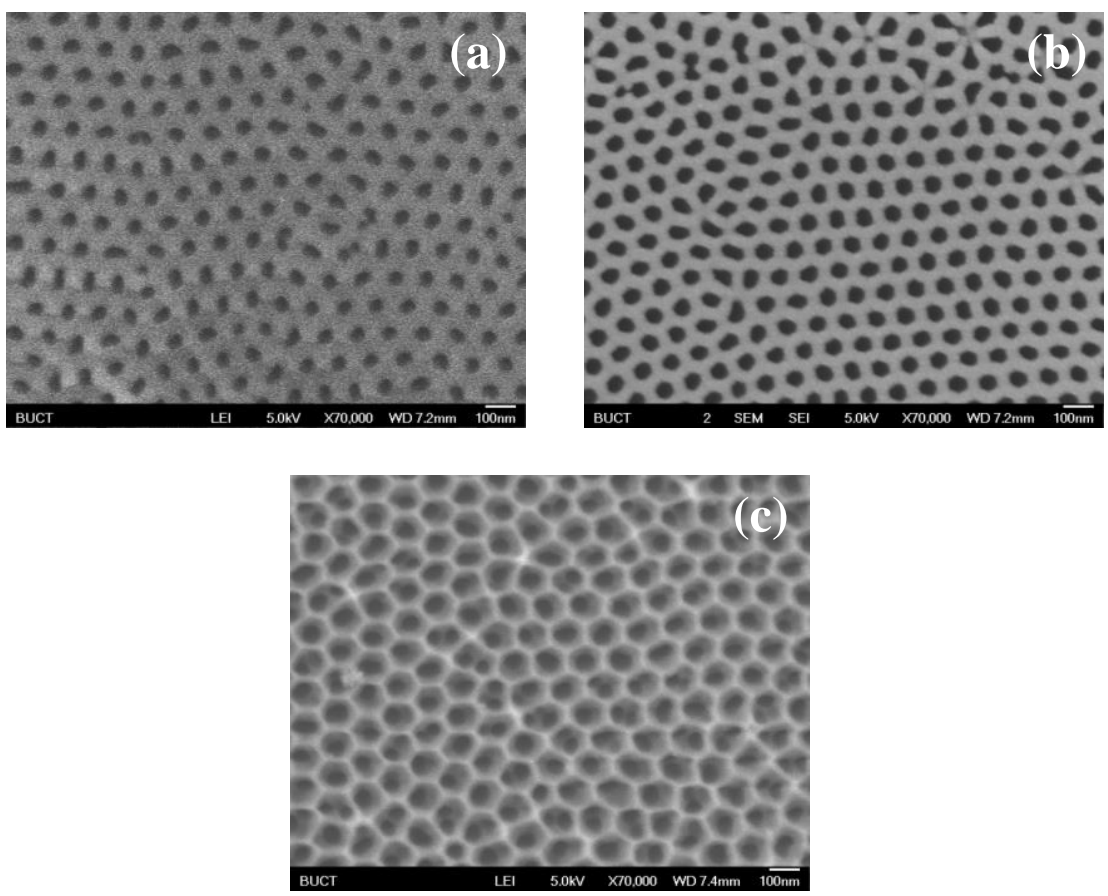

Figure S1 SEM images of the surface of AAO templates with the pore diameter of (a) 20-30 nm, (b) 40-70 nm and (c) $80-100 \mathrm{~nm}$ 

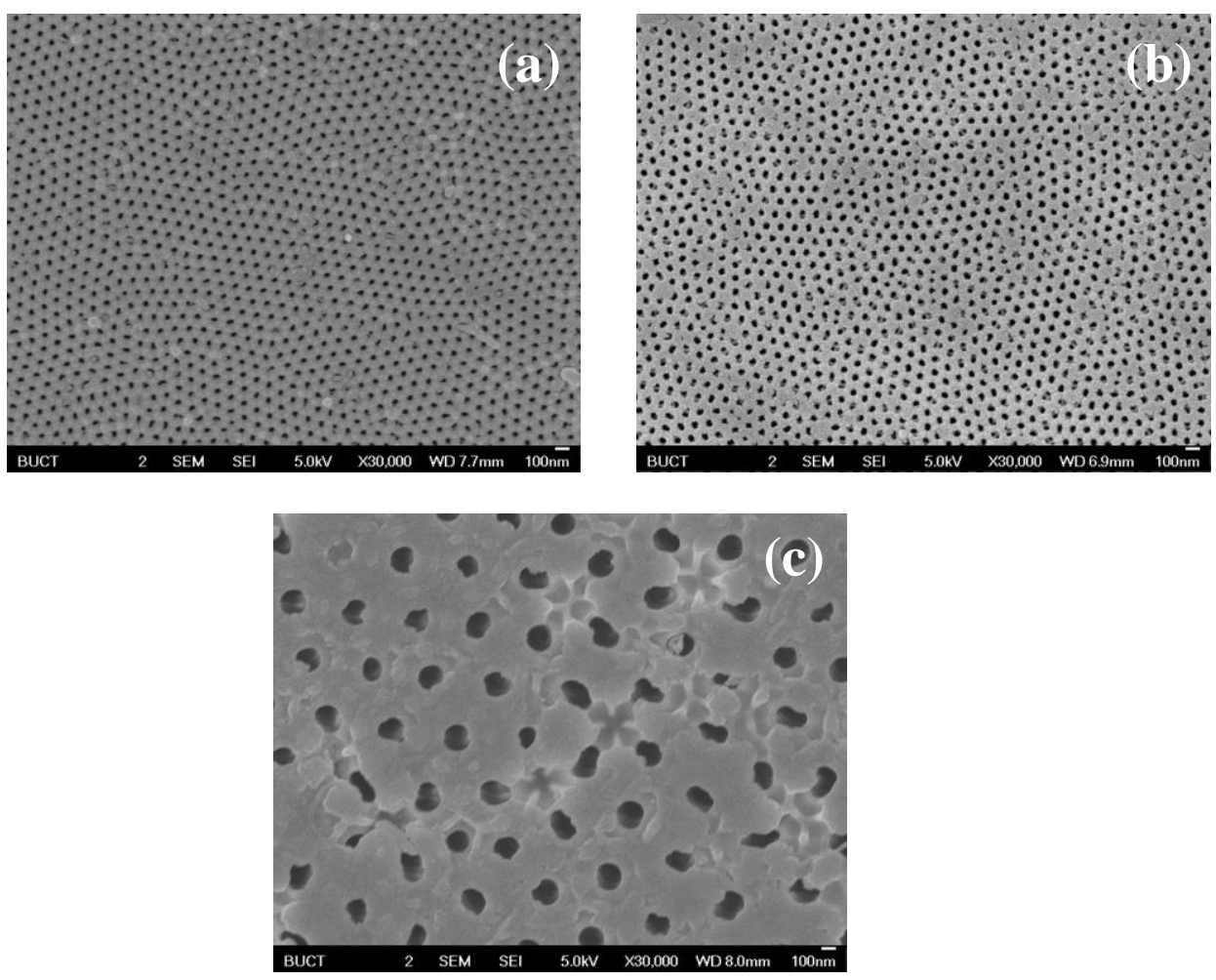

Figure S2 SEM images of the surface of AAO templates after the infiltration of PBA with the pore diameter of (a) 20-30 nm, (b) 40-70 nm and (c) 80-100 nm 


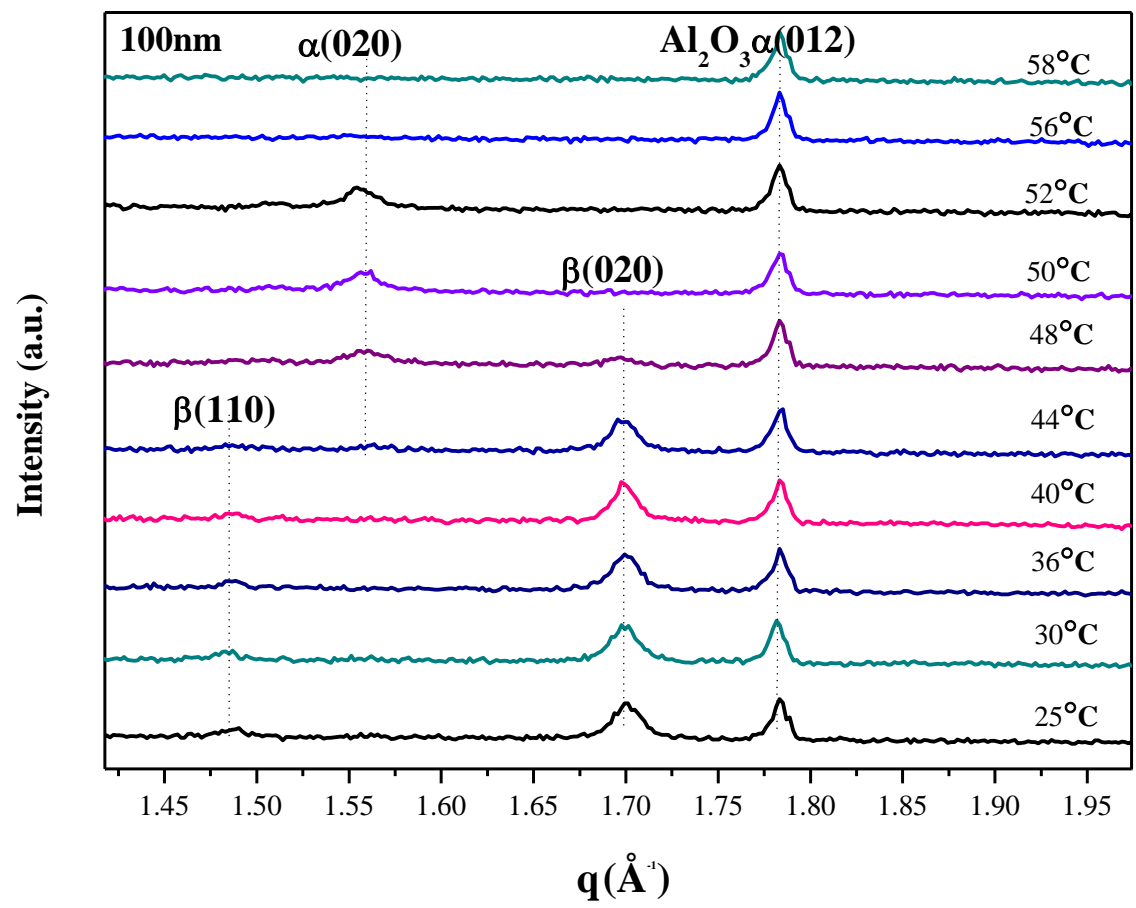

Figure S3 The WAXD profiles changes for $\beta$-PBA in the D100 with the heating rate of $1{ }^{\circ} \mathrm{C} / \mathrm{min}$. 
a

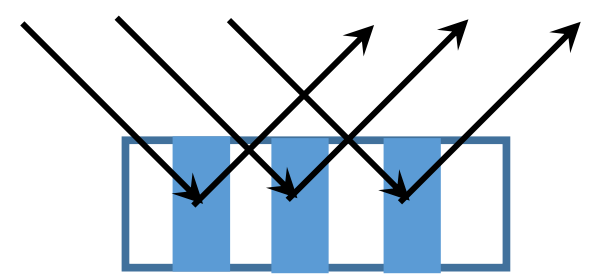

b Grazing incident mode

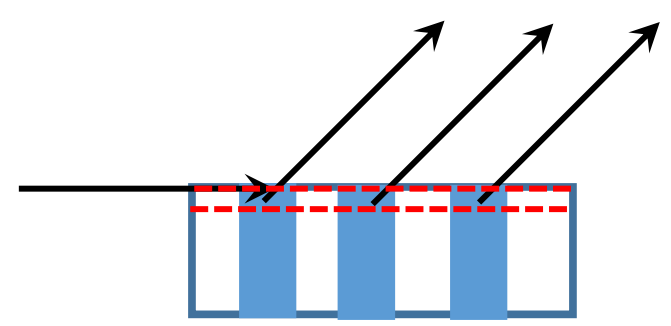

Figure S4 The sketch for the reflection mode and grazing incident mode of X-ray measurement. 